\title{
Breast cancer molecular subtypes and the metastatic microenvironment: Review of literature
}

\section{Original Article}

\author{
May Hussein Gohar \\ Department of Medical Histology and Cell Biology, Faculty of Medicine, Cairo University, \\ Cairo, Egypt
}

\begin{abstract}
Breast Cancer is a major public health concern worldwide. Breast cancer is the most common form of cancer and second leading cause of cancer death in women, with $>90 \%$ of deaths resulting from metastasis. Clinically, human breast cancers are classified into distinct molecular subtypes (luminal A/B, Her2-positive, and triple-negative [TN]) which exhibit organ-specific patterns of metastasis. The lung is one of the most common sites of metastasis in patients with aggressive triple-negative (TN) disease, while less aggressive luminal A/B cancers most often metastasize to bone. Experimentally, it has been suggested that the presence of a primary tumor may serve to induce a "pre-metastatic niche" in the lung in order to make it more hospitable for metastasizing breast cancer cells. This review integrates how the cellular and molecular components of the metastatic niche evolve in the context of molecular subtype. Understanding the cancer-induced components of the metastatic niche will develop opportunities for improved clinical management and new therapeutic strategies.
\end{abstract}

Key Words: Molecular subtypes of breast cancer, organ metastasis, premetastatic niche

Revised: 14 December 2017, Accepted: 13 February 2018

Corresponding Author: May Hussein Gohar, Department of Medical Histology and Cell Biology, Faculty of Medicine, Cairo University, Cairo, Egypt, Tel.: +201001431626, E-mail: maya.h.g@gmail.com

ISSN: $2536-9172$, Vol.1, No.2

\section{INTRODUCTION}

\subsection{Global Incidence of Breast Cancer:}

Cancer is a major public health concern worldwide. There were an estimated 14.1 million cancer cases around the world in 2012 and more than 522,000 women died worldwide due to breast cancer. In 2015, an estimated 560,000 individuals died due to breast cancer globally.
Over $90 \%$ of these cases were directly related to the metastatic spread of the disease throughout the body. The number of breast cancer cases is expected to increase to 24 million by $2035^{[1]}$. The number of cases per 100,000 women, is still lower in developing countries overall than in the West, however mortality rates from cancer are on the rise. This may be attributed to later diagnosis and poor access to treatment (Table 1$)^{[2]}$.

Table 1: Global Prevalence of breast cancer (2):

\begin{tabular}{|l|l|l|l|}
\hline & Percentage of world population & $\begin{array}{l}\text { Percentage of new breast cancer } \\
\text { cases }\end{array}$ & $\begin{array}{l}\text { Percentage of breast cancer } \\
\text { deaths }\end{array}$ \\
\hline Asia & 59 & 39 & 44 \\
\hline Africa & 15 & 8 & 12 \\
\hline United States \& Canada & 5 & 15 & 9 \\
\hline
\end{tabular}

\subsection{Breast cancer and risk factors:}

Breast cancer is a complex disease driven primarily by regulatory failure of homeostatic cellular growth mechanisms, and there are many factors that play a role in its development. Breast cancer occurrence is associated with risk factors including unmodifiable factors such as genetic inheritance, as well as modifiable factors such as obesity, stress, environmental factors, use of hormone replacement therapy, or exposure to chemicals that are known to change the breast cellular DNA (deoxyribonucleic acid) resulting in malignant transformation of normal cells ${ }^{[3]}$. This most often results in disruptions to two different categories of genes inside the nucleus: oncogenes and tumour suppressor genes. Oncogenes promote cell growth and are tumourpromoting factors when over-stimulated. Oncogenes push cells destined for apoptosis or cell death to survive and 
continue to proliferate ${ }^{[4]}$. In contrast, tumour suppressor genes are those that inhibit cell division and survival and promote apoptosis and cell cycle arrest ${ }^{[5]}$. Malignant transformation occurs either as a result of over-activation of oncogenes or down-regulation of the tumour suppressor genes and the healthy cell is transformed into a mutated malignant cell ${ }^{[6,7]}$.

The origin of breast cancer is most commonly from the ducts that carry milk or the lobules that supply them. The susceptibility of these structures to form benign and malignant transformations are in part a consequence of cycling hormonal stimulation throughout life leading to increased cell turnover and accumulation of genetic defects $^{[8,9]}$. Cancer originating from the milk ducts and lobules are referred to as ductal carcinoma or lobular carcinoma, respectively ${ }^{[10]}$. Ductal carcinoma represents the most commonly diagnosed breast tumour, accounting for approximately $75 \%$ of breast cancer $\operatorname{cases}^{[11,12]}$. Histopathological classification is commonly used when determining the stage, grade and characteristics of breast cancer. Tumours confined within the ducts or lobules that have not spread beyond their margins are referred to as either ductal carcinoma in situ (DCIS) or lobular carcinoma in situ (LCIS). Both DCIS and LCIS are non-invasive in nature and remain within their structure of origin without invading into surrounding tissues ${ }^{[13,14]}$. Therefore, when breast cancer remains in situ, traditional treatments are highly effective and successful. However, breast cancer can also spread beyond the breast area, whereby cells break off from the breast tumour and travel through the bloodstream or lymphatic system to other parts of the body, a process called metastasis. Prognosis worsens for patients with invasive ductal or lobular carcinoma; characterized by tumour that has infiltrated surrounding tissues ${ }^{[15]}$, as well as for those patients whose disease has disseminated throughout the body, leading to distant metastatic disease. Many of the basic methods for treating breast cancer often fail once the cancer has entered the metastatic phase ${ }^{[16]}$.

\subsection{Molecular Subtypes of Breast Cancer:}

Breast cancer is commonly categorized based on the molecular and genetic information of the tumor cell. However, when certain characteristics are taken into consideration, such as the hormone receptor status, the presence of HER2 (human epidermal growth factor receptor 2) and proliferation rate (Ki67), breast cancer is clinically classified into four major molecular subtypes $^{[17,18]}$. The Cancer Genome Atlas (TCGA) also classifies breast cancer into four molecular subtypes ${ }^{[19]}$. The first classification is Luminal A, which makes up 30-70\% of all breast cancers. This subtype originates in the inner (luminal) cells lining the mammary ducts. It is hormonedependent and tends to be estrogen receptor (ER)- and/or progesterone (PR)-positive, HER2-negative and displays low proliferative activity based on Ki67 expression. The second subtype is Luminal $\mathrm{B}$, which has a prevalence of $10-20 \%$ of all breast cancers and is characterized by ER/ PR-positivity, high Ki67, and/or HER2-positivity. Patients with Luminal A/B breast cancer have the best prognosis, and if they do progress to metastasis is usually to the bone $^{[17]}$. The third subtype HER2-enriched, with ER/PR negativity and positive HER2, with a prevalence of 5-15\% of all breast cancers. The fourth type is the triple negative/ basal-like (ER-/PR-/HER2-), with a prevalence of 15-20\% of all breast cancers. The latter two subtypes (TN) are basallike tumours, due to the tumor cells expressing similar features to those of the outer (basal) cells surrounding the mammary ducts. Triple negative (TN) breast tumors have the highest incidence of metastasis and show a high rate of metastasis to the lungs. The TN tumors are often more aggressive and have a poorer prognosis (at least within the first five years after diagnosis) compared to the ERpositive subtypes (Luminal A and Luminal B tumors) ${ }^{[20]}$.

\subsection{The Metastatic Cascade and Breast Cancer:}

Metastasis is a complex process by which tumour cells spread to distant sites throughout the body. This occurs by a series of coordinated cellular events: (A) development of a primary tumour; (B) tumour cells from the primary tumour escape, invading (intravasating) into the vasculature; $(\mathrm{C})$ tumour cells disseminate through the blood and/or lymphatics, until they arrest in the capillary beds of distant organs and extravasate into a new tissue where they can ultimately initiate and establish a secondary tumour, distant from the primary tumour ${ }^{[21]}$.

Current therapies are largely non-curative in the metastatic setting ${ }^{[22]}$. Lung metastasis often occurs within 5 years of initial breast cancer diagnosis and has a significant impact on patient morbidity and mortality ${ }^{[23]}$. Physiologically, these metastases disrupt normal lung function, and result in coughing, labored breathing, hemoptysis, and eventual death ${ }^{[24,25]}$. Advanced lung metastases remain difficult to treat, highlighting the need to better understand the cellular and molecular drivers in order to develop new therapeutic strategies.

\subsection{Metastatic Organ Tropism and the Pre-}

\section{Metastatic Niche:}

Breast cancer has special preference to metastasize to specific organs, which is termed "organ tropism"[26,27]. Multiple theories have been developed to explain the process of organ-specific tropism, most notable of which are Stephen Paget's seminal "seed and soil" hypothesis in $1889^{[28]}$, and Ewing's mechanical arrest theory ${ }^{[29]}$. The "seed and soil" theory states that metastasis selectively colonizes in specific organs because of a "match" between the migrating tumor cell and a "suitable" environment. However, Ewing's theory, anticipated thirty years later, that organ tropism can be accounted for by circulatory patterns within the body, when the cancer cells are mechanically arrested in the first capillary bed they meet. Dr. Leonard Weiss, who compared both theories in an autopsy study examining the frequency of metastasis in a number of anatomic sites, leans towards a combination of the two theories being correct ${ }^{[30]}$. 
It is possible that these two theories are not equally exclusive, cells arrest due to mechanical obstruction and/ or specific chemical signals and then require a suitable microenvironment for initiation and maintenance of secondary tumor growth ${ }^{[27]}$.

More recently, an additional layer of complexity has been uncovered whereby the presence of a primary tumour may actually promote that development of a "pre-metastatic niche" in distant organs before the metastatic cells actually arrive in the secondary site. The pre-metastatic niche is best explained as a supportive and receptive tissue microenvironment undergoing a series of molecular and cellular changes to build the fertile soil for tumour settlement and metastasis in distant organs ${ }^{[31,32]}$.

\subsection{Molecular Aspects of the Pre-Metastatic Niche:}

Figure 1 illustrates the role and steps of the premetastatic niche in the promotion of tumour metastasis, including the molecular and cellular components of premetastatic niche formation and the pathological sequence of events. The establishment of a pre-metastatic niche passes through the following phases until it forms the macrometastases in the target organ:

\section{A. Priming:}

The primary tumour undergoes uncontrolled proliferation and become hypoxic and inflammatory. This results in release of growth factors including vascular endothelial growth factor (VEGF), placental growth factor (PlGF), transforming growth factors (TGF), and other molecular components. As a result, inflammatory S100 chemokines are upregulated in pre-metastatic sites, leading to clustering of bone marrow-derived hematopoietic progenitor cells (HPCs) which can induce the mobilization of bone marrow-derived cells (BMDCs) ${ }^{[33,34]}$

\section{B. Licensing Phase:}

This is the key link between the maturation of the premetastatic niche and initiation of tumour metastasis. The BMDCs and immune cells are continuously mobilized and recruited into the secondary sites ${ }^{[35]}$.

\section{Initiation Phase:}

In this phase the pre-metastatic niche contributes to initiation of metastasis by facilitating the extravasation of circulating tumour cells (CTCs) from the vasculature and attracting tumour cell colonization into the niche. Mobilization of platelet-deployed stromal-derived growth factor 1 (SDF-1) from bone marrow to the premetastatic niche, also attracts $\mathrm{C}-\mathrm{X}-\mathrm{C}$ chemokine receptor type $4\left(\mathrm{CXCR} 4^{+}\right)$haematopoetic progenitor cells (HPCs) and metastatic tumour cells (MTCs) ${ }^{[36]}$. HPCs secrete a variety of pre-metastatic factors including TNF- $\alpha$, matrix metalloproteinase 9 (MMP-9) and TGF- $\beta$. Activated fibroblasts secrete fibronectin, an important adhesion protein in the niche. The upregulation of integrins, TNF- $\alpha$, fibronectin, and MMPs enhances the adhesion of MTCs to the pre-metastatic niche ${ }^{[37]}$. The pre-metastatic niche also regulates tumour dormancy between the MTCs and reactivates them when the pre-metastatic niche is ready and suitable for the growth of the tumour. The well-established pre-metastatic niche is then competent for the initiation and formation of micrometastases ${ }^{[38]}$.

\section{Progression Phase:}

In this phase the MTCs proliferate, and expansion of the tumour mass facilitates the change in micometastases to macometastases. The recruitment of endothelial progenitor cells (EPCs) to the early metastatic niche mediates the angiogenic switch and enables progression to macrometastases ${ }^{[31,39]}$.

In summary, tumor-type specific factors are released from the primary tumor to facilitate changes in the microenvironment of distant sites before the cells arrive, initiating a so-called "pre-metastatic niche". Cancer cells and associated stromal cells secrete chemokines that direct the migration, proliferation and differentiation of the vascular cell network to support the tumor and the metastatic microenvironment. It has been demonstrated that bone marrow-derived cells respond to these systemic factors by migrating to the pre-metastatic niche and forming clusters of cells in the tissue parenchyma at the sites of metastasis before evidence of any primary tumor cells $^{[28]}$. CD117 is an important cell surface marker used to identify certain types of hematopoietic (blood) progenitors in the bone marrow. More specifically, hematopoietic stem cells (HSC), multipotent progenitors (MPP), and common myeloid progenitors (CMP) express high levels of CD117 . Common lymphoid progenitors (CLP) express low surface levels of CD117. Signaling through CD117 plays a role in cell survival, proliferation, and differentiation. CD117 is a proto-oncogene, meaning that overexpression or mutations of this protein can lead to cancer. Primary tumour-derived components, tumour-mobilized bone marrow-derived cells (BMDC) with their surface markers like CD117, and the local stromal microenvironment of the host are the three major factors crucial for the formation of the premetastatic niche ${ }^{[40]}$. These are the three factors which need to be investigated in order to gain a greater understanding of the pre-metastatic niche in the lung during breast cancer progression with the goal of new and improved therapeutic strategies.

\subsection{Criteria for Establishment of a Pre-Metastatic}

\section{Niche (Tumour Microenvironment):}

The tumour microenvironment is composed mainly of endothelial cells, fibroblasts, perivascular cells, and inflammatory cells, which regulate the tumorigenic process. There are six characteristics that are believed to determine whether metastatic tumour cells may die, survive and colonize, or become dormant after arrival in the secondary organ. These are:

1. Immunosuppression: Regulatory immune cells like CD8+ T cells, natural killer cells and non-classical monocytes inhibit local tumour immunity and contribute to 
the formation of immunosuppressive pre-metastatic niche. However, some regulatory immunosuppressive cells such as MDSCs, macrophages, and Treg cells within the premetastatic niche potentially suppress anti-tumour immune responses ${ }^{[41]}$.

2. Chronic Inflammation triggers many signaling pathways and molecular components which are important drivers of tumour development and metastasis. An example of this is the pro-inflammatory mediators S100A8/A9 become upregulated in the lung pre-metastatic niche. This in turn induces the expression of serum amyloid A that recruits myeloid cells to these sites. These myeloid cells will enhance pre-metastatic niche formation in an inflammatory state and promote migration of the of the primary tumour cells to the secondary lung site. Clara cells of the lungs are also involved in the inflammatory process, and $\mathrm{CD} 11 \mathrm{~b}^{+}$cells in the lung pre-metastatic niche enhances the inflammatory and proliferative processes occurring in the lung and stimulate the expression of MMPs to enhance the adhesion ${ }^{[42]}$.

3. Angiogenesis and Vascular Permeability: Both angiogenesis and vascular permeability become increased in the pre-metastatic niche to promote metastasis. The migrated bone marrow monocytes and the endothelial progenitor cells express high levels of VEGF and other proangiogenic factors, which help facilitate the switch from micrometastasis to macrometastasis ${ }^{[43]}$. In addition, the increased vascular permeability within the pre-metastatic niche will facilitate the extravasation of circulating tumour cells and the establishment of lung metastasis ${ }^{[4]]}$.

4. Lymphangiogenisis: The lymphatic system and lymphangiogenesis often serve as the initial route of tumour dissemination $^{[45]}$, and clinical research has confirmed that tumour-derived VEGF-A and VEGF-D induce prometastatic lymphangiogenisis ${ }^{[46]}$.

5. Organ Tropism: Primary breast cancer cell-derived VEGF alters the pre-metastatic lung microenviroment by triggering an inflammatory response and prostaglandin production, which influences the preferential migration of the cancer cells to the lung ${ }^{[47]}$.

6. Metabolic, Stromal and Epigenetic Reprogramming: These processes are involved in pre-metastatic nichepromoted tumour metastasis. Breast cancer cells can suppress glucose uptake by non-tumour cells in the premetastatic niche, by secreting vesicles that carry high levels of the miR-122 microRNA. High miR-122 levels in the circulation have been associated with metastasis in breast cancer patients, and Fung et al showed that cancercell-secreted miR-122 facilitates metastasis by increasing nutrient availability in the pre-metastatic niche ${ }^{[48]}$.

\subsection{The Soluble Lung Microenvironment:}

Previous research studies have previously employed novel ex vivo model systems of the lung and has demonstrated that soluble and matrix factors in the normal (healthy) lung microenvironment can interact with aggressive breast cancer cells to promote metastatic behavior in the lung ${ }^{[49]}$. Organs representing common sites of breast cancer metastasis (lung, bone, liver, brain, $\mathrm{LN}$ ) are isolated from healthy mice and used to generate organ-conditioned media (CM). Using this model, Chu et al (2014) demonstrated that different human breast cancer cell lines show specific chemotactic and proliferative behaviours in response to various organ-CM, reflective of their metastatic behaviours in vivo ${ }^{[50]}$. Specifically, the most aggressive of the cell lines, MDA-MB-231, showed increased migration patterns towards bone, lymph node, and lung-CM. The second most aggressive cell line, SUM159, displayed enhanced migration towards the bone, brain, LN, and while the two least aggressive cells lines (SUM149 and MDA-MB-468) demonstrated increased migration to lung- $\mathrm{CM}$ only. In addition to increased migratory patterns, MDA-MB-231 and MDAMB-468 cell lines demonstrated cell line specific patterns of proliferation in response to organ-CM. MDA-MB-231 cells showed increased proliferation in the presence of liver and lung-CM and MDA-MB-468 cells showed increased proliferation in the presence of lung- $\mathrm{CM}^{[50]}$. These results indicate the potential of the lung microenvironment for promoting metastatic progression of breast cancer cells.

Protein array analysis of organ-CM identified several secreted metastasis-promoting factors from the lung and the innovative $3 \mathrm{D}$ ex vivo pulmonary metastasis assay (PuMA) confirmed the lung as an effective metastatic microenvironment ${ }^{[51]}$.

\subsection{The Insoluble Lung Microenvironment:}

The cellular part of the lung is composed of over 60 different cell types involved in various functions including sensory, mechanical, secretory and transport. The noncellular part of the lung or the extracellular matrix (ECM) constitute about $15 \%$ of alveolar tissue and $50 \%$ of nonalveolar tissue ${ }^{[52]}$. The extracellular matrix is a dynamic 3D structure composed of a myriad of structural proteins including collagen and elastin, specialized proteins such as fibronectin and laminin, as well as high- molecular weight proteoglycans, which function to support surrounding parenchymal cells $\mathrm{s}^{[53]}$. Other extracellular matrix components include Tenascin $\mathrm{C}$, which is an oligomeric glycoprotein composed of individual polypeptides with molecular weights ranging from 180 to $\sim 300 \mathrm{kDa}$. These protein modules are lined up like beads on a string and give rise to long and extended molecules ${ }^{[54]}$. Tenascin-C has been shown to interact with fibronectin in order to modify cell adhesion ${ }^{[55]}$. A solid-state interaction between fibronectin and $\mathrm{TN}-\mathrm{C}$ results in cellular up-regulation of matrix metalloproteinase expression ${ }^{[56]}$.

Periostin is a secreted extracellular matrix protein that was originally identified in cells from the mesenchymal lineage. It binds to several integrin receptors. These receptors have been demonstrated to have function in cell adhesion, migration, and survival in these cells. In many cancers, periostin binds to integrins on cancer cells, activating the signaling pathways. This leads to increased 
cell survival, invasion, angiogenesis, metastasis, and the epithelial-mesenchymal transition ${ }^{[57]}$. Many types of epithelial and endothelial cells are dependent upon adhesion to the ECM for their continued survival and often undergo apoptosis when this adhesion is disrupted. Although cancer cells are characterized by their unique ability to progress and grow in the absence of ECM adhesion, solid tumours often exist in a dynamic relationship between anchoragedependence and independence ${ }^{[53]}$.

Fig. 1: Figure is modified from Kaplan et al., 2005 $5^{[31]}$ and Lyden et al., 2009 $9^{[35]}$.

\section{Molecular aspects of the premetastatic niche}

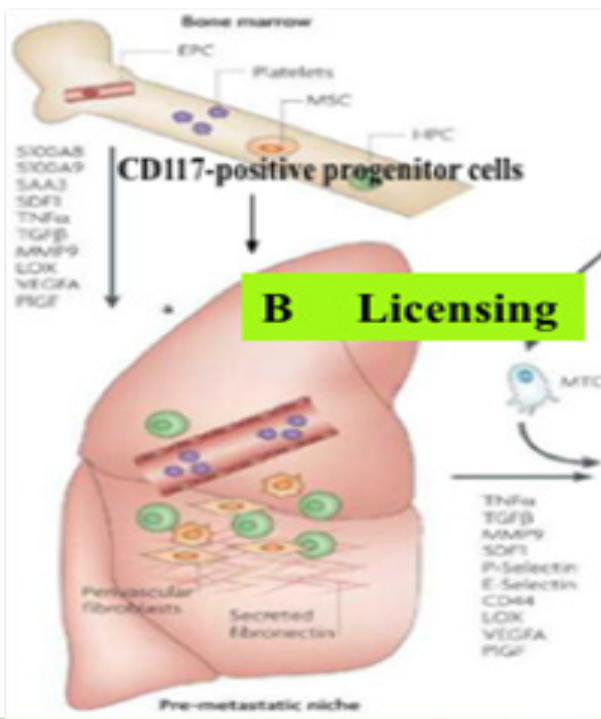

1. Chemokines

2. Activated fibroblasts

3. Stromal fibronectin

4. VEGFR1 myeloid progenitor cells
A Priming



1. Integrins

2. MTC

3. TNF alpha

4. MatrixMetalloproteinases MMP
Growth factersasveG. placental grewth facter. trasuforming zrowth factor trans



1. Bone marrow endothelial progenitor cells

2. Stromal cells e.g. fibroblasts

3. Tumour associated myeloid cells

(A) In the priming phase, primary tumor cells produce various soluble factors molecular components, to trigger the formation of an immature pre-metastatic niche in the secondary organ site by up-regulation of specific chemokines. (B) In the licensing phase, sequestration of bone marrow-derived cells like the VEGFR1, CD-117 progenitor cells and other regulatory/suppressive immune cells are mobilized and recruited into the secondary sites (lungs) in response to tumor-derived molecular components. Also activation of fibroblasts and secretion of fibronectin starts in the premetastatic niche. (C) In the initiation phase, metastatic tumour cells (MTA) arrive and colonize at the fertile pre-metastatic niche, increased expression of integrins, TNF alpha and Matrix-Metalloproteins (MMP) resulting in micrometastases. (D) In the progression phase, the pre-metastatic niche can host more migrated tumor cells, more stroma cells and the migrated Bone marrow endothelial progenitor cells express new blood vessels, leading to macrometastases.

\section{CONCLUSION}

As metastasis is the major cause of death from cancer, investigating the sources of metastatic disease and investigating the components of the metastatic microenvironment will lead to develop therapies to be used clinically. As the lung is one of the major deadly sites of breast cancer metastasis, especially in patients with aggressive "triple negative" Breast cancer, so understanding the cancer-induced components of the metastatic niche will develop opportunities for improved clinical management and new therapeutic strategies.

\section{CONFLICT OF INTEREST}

There are no conflicts of interest.

\section{REFERENCES}

1. Ferlay, J., Soerjomataram, I., Dikshit, R., Eser, S., Mathers, C., Rebelo, M., \& Bray, F. (2015). Cancer incidence and mortality worldwide: sources, methods and major patterns in GLOBOCAN 2012. International Journal of Cancer, 136(5), E359-86.

2. Bray, F., Ren, J. S., Masuyer, E., \& Ferlay, J. (2013). Global estimates of cancer prevalence for 27 sites in 
the adult population in 2008. International Journal of Cancer, 132(5), 1133-1145.

3. Willett, W.C., Tamimi, R., Hankinson, S.E., Hazra, A, Eliassen, A.H., Colditz, G.A. Chapter 18: Nongenetic Factors in the Causation of Breast Cancer, in Harris JR, Lippman ME, Morrow M, Osborne CK. Diseases of the Breast, 5th edition, Lippincott Williams \& Wilkins, (2014), 1224p.

4. Croce, C.M.: Oncogenes and cancer. The New England journal of medicine (2008), 358(5):502-511.

5. Sherr, C.J.: Principles of tumor suppression. Cell 2004, 116(2):235-246.

6. Knudson, A.G.: Two genetic hits (more or less) to cancer. Nature reviews Cancer (2001), 1(2):157-162.

7. Baylin, S.B., Ohm, J.E.: Epigenetic gene silencing in cancer - a mechanism for early oncogenic pathway addiction? Nature reviews Cancer (2006), 6(2): 107 116.

8. Fata, J.E., Werb, Z., Bissell, M.J.: Regulation of mammary gland branching morphogenesis by the extracellular matrix and its remodeling enzymes. Breast cancer research: BCR (2004), 6(1):1-11.

9. Sternlicht, M.D.: Key stages in mammary gland development: the cues that regulate ductal branching morphogenesis. Breast cancer research: BCR (2006), 8(1):201

10. Sariego, J.: Breast cancer in the young patient. The American surgeon (2010), 76(12):1397-1400.

11. Li, C.I., Uribe, D.J., Daling, J.R.: Clinical characteristics of different histologic types of breast cancer. Br J Cancer (2005), 93(9):1046-1052.

12. Stopeck, A.T.C., P.; Thompson, P.A.: Breast Cancer Pathophysiology. In.: Medscape; (2015).

13. Sakorafas, G.H., Tsiotou, A.G.: Ductal carcinoma in situ (DCIS) of the breast: evolving perspectives. Cancer Treat Rev (2000), 26(2):103-125.

14. Takahashi, K., Saito, M., Makita, M., Tada, T., Uchida,Y., Yoshimoto, M., Kasumi, F., Akiyama, F., Sakamoto, G.: Surgery for ductal carcinoma in situ. Breast Cancer (2000), 7(4):337-340.

15. Simpson, P.T., Gale, T., Fulford, L.G., Reis-Filho, J.S., Lakhani, S.R.: The diagnosis and management of preinvasive breast disease: pathology of atypical lobular hyperplasia and lobular carcinoma in situ. Breast cancer research: BCR (2003), 5(5):258-262.
16. Chambers, A.F., Groom, A.C., MacDonald, I.C.: Dissemination and growth of cancer cells in metastatic sites. Nature reviews Cancer (2002), 2(8):563-572.

17. Kennecke, H., Yerushalmi, R., Woods, R., Cheang, M.C., Voduc, D., Speers, C.H., Nielsen, T.O., Gelmon, K.: Metastatic behavior of breast cancer subtypes. Journal of clinical oncology: official journal of the American Society of Clinical Oncology (2010), 28(20):3271-3277.

18. Voduc, K.D., Cheang, M.C., Tyldesley, S., Gelmon, K., Nielsen, T.O., Kennecke, H.: Breast cancer subtypes and the risk of local and regional relapse. Journal of clinical oncology: official journal of the American Society of Clinical Oncology (2010), 28(10):16841691.

19. Cancer Genome Atlas N: Comprehensive molecular portraits of human breast tumours. Nature (2012), 490(7418):61-70.

20. Carey, L.A., Perou,C.M., Livasy, C.A., Dressler, L.G., Cowan, D., Conway, K., Karaca, G., Troester MA, Tse CK, Edmiston S, Deming SL, Geradts J, Cheang MC, Nielsen TO, Moorman PG, Earp HS, Millikan $\mathrm{RC}$ : Race, breast cancer subtypes, and survival in the Carolina Breast Cancer Study. JAMA (2006), 295(21):2492-2502.

21. Minn, A.J., G.P. Gupta, P.M. Siegel, P.D. Bos, W. Shu, D.D. Giri, A. Viale, A.B. Olshen, W.L. Gerald, and J. Massague, Genes that mediate breast cancer metastasis to lung. (2005), Nature. 436(7050): 518-24.

22. Kreisman, H., N. Wolkove, H.S. Finkelstein, C. Cohen, R. Margolese, and H. Frank, Breast cancer and thoracic metastases: review of 119 patients. (1983), Thorax. 38(3): 175- 9.

23. Rashid, O.M. and K. Takabe, The evolution of the role of surgery in the management of breast cancer lung metastasis. 2012, J Thorac Dis. 4(4): 420-4.

24. Hellman, S. and R.R. Weichselbaum, Oligometastases. (1995), J Clin Oncol. 13(1): 8-10.

25. Fidler IJ: The organ microenvironment and cancer metastasis. Differentiation; research in biological diversity (2002), 70(9-10):498-505.

26. Nguyen DX, Bos PD, Massague J: Metastasis: from dissemination to organ- specific colonization. Nature reviews Cancer (2009), 9(4):274-284.

27. Chu JE, Allan AL: The Role of Cancer Stem Cells in the Organ Tropism of Breast Cancer Metastasis: A Mechanistic Balance between the "Seed" and the 
"Soil"? International journal of breast cancer (2012): Article ID 209748: 1-12.

28. Paget S. The distribution of secondary growths in cancer of the breast. 1889. Cancer and Metastasis Reviews. (1989); 8(2):98-101.

29. Ewing J. A Treatise on Tumors. Philadelphia, Pa, USA: W.B. Saunders Company; 1928. Neoplastic Diseases, a text-book on tumours, Philadelphia and London, W. B. Saunders company, (1919).

30. Weiss, L. (1992) "Comments on hematogenous metastatic patterns in humans as revealed by autopsy," Clinical and Experimental Metastasis, vol. 10, no. 3, pp. 191-199.

31. Kaplan, R.N., Riba, R.D., Zacharoulis, S., Bramley, A.H., Vincent, L., Costa, C., MacDonald, D.D., Jin, D.K., Shido, K., Kerns, S.A., et al. (2005). VEGFR1positive hematopoietic bone marrow progenitors initiate the pre-metastatic niche. Nature 438, 820-827.

32. Psaila, B., Kaplan, R. N., Port, E. R. \& Lyden, D. (2006) Priming the 'soil' for breast cancer metastasis: the pre- metastatic niche. Breast Dis. 26, 65-74.

33. Sleeman, J.P. (2012). The metastatic niche and stromal progression. Cancer Metastasis Rev. 31, 429-440.

34. Chin, A.R., and Wang, S.E. (2016). Cancer tills the premetastatic field: mech- anistic basis and clinical implications. Clin. Cancer Res. 22, 3725-3733.

35. B. Psaila and David Lyden (2009). The metastatic niche: Adapting the foreign soil. Nat Rev Cancer. 2009 April;9 (4):285-293.

36. Jin, D. K., Shido, K., Kopp, H.G., Petit, I., Shmelkov, S.V., Young, L.M., Hooper, A.T., Amano, H., Avecilla, S.T., Heissig, B., Hattori, K., Zhang, F., Hicklin, D.J., Wu, Y., Zhu, Z., Dunn, A., Salari, H., Werb, Z., Hackett, N.R., Crystal, R.G., Lyden, D., Rafii, S. (2006). Cytokine-mediated deployment of SDF-1 induces revascularization through recruitment of CXCR4+ hemangiocytes. Nature Medicine, 12(5), $557-567$.

37. Yamamura, Y., Asai, N., Enomoto, A., Kato, T., Mii, S., Kondo, Y., Ushida, K., Niimi, K., Tsunoda, N., Nagino, M., et al. (2015). Akt-Girdin signaling in cancer- associated fibroblasts contributes to tumor progression. Cancer Res. 75, 813-823.

38. Romero, I., Garrido, F., and Garcia-Lora, A.M. (2014). Metastases in immune- mediated dormancy: a new opportunity for targeting cancer. Cancer Res. 74, $6750-6757$.
39. Gao D, et al. Endothelial progenitor cells control the angiogenic switch in mouse lung metastasis. Science. (2008); 319:195-8.

40. Edling, C. E., \& Hallberg, B. (2007). c-Kit-A hematopoietic cell essential receptor tyrosine kinase. International Journal of Biochemistry and Cell Biology, 39, 1995-1998.

41. Liu, Y., and Cao, X. (2016). Immunosuppressive cells in tumor immune escape and metastasis. J. Mol. Med. 94, 509-522.

42. Wculek, S.K., and Malanchi, I. (2015). Neutrophils support lung colonization of metastasis-initiating breast cancer cells. Nature 528, 413-417.

43. Saharinen, P., Eklund, L., Pulkki, K., Bono, P., and Alitalo, K. (2011). VEGF and angiopoietin signaling in tumor angiogenesis and metastasis. Trends. Mol. Med. 17, 347-362.

44. Huang, Y., Song, N., Ding, Y., Yuan, S., Li, X., Cai, H., Shi, H., and Luo, Y. (2009). Pulmonary vascular destabilization in the premetastatic phase facili- tates lung metastasis. Cancer Res. 69, 7529-7537.

45. Karnezis, T., Shayan, R., Caesar, C., Roufail, S., Harris, N.C., Ardipradja, K., Zhang, Y.F., Williams, S.P., Farnsworth, R.H., Chai, M.G., et al. (2012). VEGF-D promotes tumor metastasis by regulating prostaglandins produced by the collecting lymphatic endothelium. Cancer Cell 21, 181-195.

46. Wakisaka, N., Hasegawa, Y., Yoshimoto, S., Miura K., Shiotani, A., Yo- koyama, J., Sugasawa, M., Moriyama-Kita, M., Endo, K., and Yoshizaki, T. (2015). Primary tumor-secreted lymphangiogenic factors induce pre-metasta- tic lymphvascular niche formation at sentinel lymph nodes in oral squamous cell carcinoma. PLoS One 10, e0144056.

47. Liu, S., Jiang, M., Zhao, Q., Li, S., Peng, Y., Zhang, P., and Han, M. (2014). Vascular endothelial growth factor plays a critical role in the formation of the premetastatic niche via prostaglandin E2. Oncol. Rep. 32, $2477-2484$

48. Fong, M.Y., Zhou, W., Liu, L., Alontaga, A.Y., Chandra, M., Ashby, J., Chow, A., O’Connor, S.T., Li, S., Chin, A.R., et al. (2015). Breast-cancersecreted miR-122 reprograms glucose metabolism in premetastatic niche to promote metastasis. Nat. Cell. Biol. 17, 183-194.

49. Piaseczny, M.M., D. Goodale, and A.L. Allan. The lung microenvironment influences the metastatic behavior of breast cancer cells in an innovative 3D ex 
vivo pulmonary metastasis model. (2015), Cancer Res (75 (Suppl): 2379.

50. Chu, J.E., Y. Xia, B. Chin-Yee, D. Goodale, A.K. Croker, and A.A. L., Lung-derived factors mediate breast cancer cell migration via CD44 receptor-ligand interactions in a novel ex vivo system for analysis of organ-specific soluble proteins. (2014), Neoplasia. 16(2): 180-191

51. Mendoza, A., S.H. Hong, T. Osborne, M.A. Khan, K. Campbell, J. Briggs, A. Eleswarapu, L. Buquo, L. Ren, S.M. Hewitt, H. Dakir el, S. Garfield, R. Walker, G. Merlino, J.E. Green, K.W. Hunter, L.M. Wakefield, and C. Khanna, Modeling metastasis biology and therapy in real time in the mouse lung. (2010), J Clin Invest. 120(8): 2979-88.

52. Franks TJ, Colby TV, Travis WD, Tuder RM, Reynolds HY, Brody AR, Cardoso WV, Crystal RG, Drake CJ, Engelhardt J, Frid M, Herzog E, Mason R, Phan SH, Randell SH, Rose MC, Stevens T, Serge J, Sunday ME, Voynow JA, Weinstein BM, Whitsett J, Williams MC: Resident cellular components of the human lung: current knowledge and goals for research on cell phenotyping and function. Proceedings of the American Thoracic Society (2008), 5(7): 763-766.

53. Rintoul RC, Sethi T: The role of extracellular matrix in small-cell lung cancer. Lancet Oncol (2001), 2(7):437442 .

54. Gulcher JR, Nies DE, Alexakos MJ, Ravikant NA, Sturgill ME, Marton LS, Stefansson K (1991). "Structure of the human hexabrachion (tenascin) gene". Proc. Natl. Acad. Sci. U.S.A. 88 (21): 9438-42.

55. Chung CY, Zardi L, Erickson HP (1995). "Binding of Tenascin-C to soluble fibronectin and matrix fibrils". J. Biol. Chem. 270 (48): 29012-7.

56. Tremble P, Chiquet-Ehrismann R, Werb Z (1994). "The extracellular matrix ligands fibronectin and tenascin collaborate in regulating collagenase gene expression in fibroblasts". Mol. Biol. Cell. 5 (4): 439-53

57. Morra L, Moch H (Nov 2011). "Periostin expression and epithelial-mesenchymal transition in cancer: a review and an update". Virchows Archiv. 459 (5): 465-75. 